\title{
Hybrid Rail Vehicles using Digital Hydraulics
}

\author{
W.H.S. Rampen ${ }^{1}$, G.P. Voller ${ }^{2}$, S.M. Laird ${ }^{2}$, D. Abrahams ${ }^{2}$ \\ and J. Macpherson ${ }^{2}$
}

\author{
${ }^{1}$ The University of Edinburgh, Edinburgh, United Kingdom \\ ${ }^{2}$ Artemis Intelligent Power Ltd, Edinburgh, United Kingdom
}

\begin{abstract}
This paper shows how a new generation of digital hydraulic machines can be used to create a series hybrid transmission for a diesel-powered railcar, with gas accumulators used for on-board energy storage. Simulation results are provided for a concept passenger vehicle based on this technology, showing improvements to performance and fuel consumption. The conversion of a demonstration vehicle is described and test results are presented.
\end{abstract}

Keywords: hybrid, powertrain, hydrostatic, hydraulic, railcar, transmission.

\section{Introduction}

Whilst electrified railways are increasing throughout the developed world, there still remains a very large fraction of routes which remain unserved. For many of them the volume of traffic is insufficient to justify the heavy expense of electric infrastructure. The self-propelled diesel railcar, or Diesel Multiple Unit (DMU), looks to be with us for some time yet. At present in the United Kingdom there are approximately 2,500 DMU carriages, operating on a network which is only $45 \%$ electrified. In the rest of the world there are at least six times this number. Many of the rail networks in developing countries have no electrified routes at all and so could constitute a growing market for these trains.

Although various hybrid architectures are being tried to replace the DMU - using combinations of hydrogen and batteries as substitutes for liquid fuels - it would seem that the low energy density and cost of these solutions makes them poor competitors to existing prime-movers which use liquid fuels, at least in terms of current economics. Further, in the case of hydrogen, the energy losses in the journey from electricity to motive wheel power seem to be challenging. Additionally, hydrogen is very difficult to transport, given that liquefied hydrogen is cryogenic and presents many technical challenges. Batteries may one day attain the energy and power 
densities needed to provide the required range of a typical route, but this is still some way off. The time needed to charge, or change, them is also a problem that arises with continuous usage. Cignini et al. [1] have studied the use of batteries and supercapacitors in a public transport vehicle and examined these issues.

Modern diesel engines are much cleaner than those in typical DMUs - many of which are by now decades old and built under very different regulations. The real problem with the continued use of diesel is the carbon dioxide emissions and their contribution to climate change. The solution suggested in this paper does not eliminate $\mathrm{CO}_{2}$ but it could significantly reduce it, using technology that is available now. Whilst this might only be seen as a short-term solution, there has been much work done on alternative synthetic fuels - such as di-methyl ether or DME and eDiesel - both of which can originate from solar energy. Arguably a fuel cycle such as this could be made carbon neutral, whilst still making use of the vast global existing infrastructure. As such it could be seen as part of a decarbonised future transport system.

At present much of the fuel used in these DMUs ends up as heat. On a typical commuter route about half of the total energy emerging from the engine ends up as heat in the brakes. Another quarter is lost in the transmission. Further waste heat is evolved in driving the cooling fans and the electric alternator, see Wheals [2]. The individual energy uses, measured on a Class 158 carriage on Scottish lowland routes are shown in Figure 1.
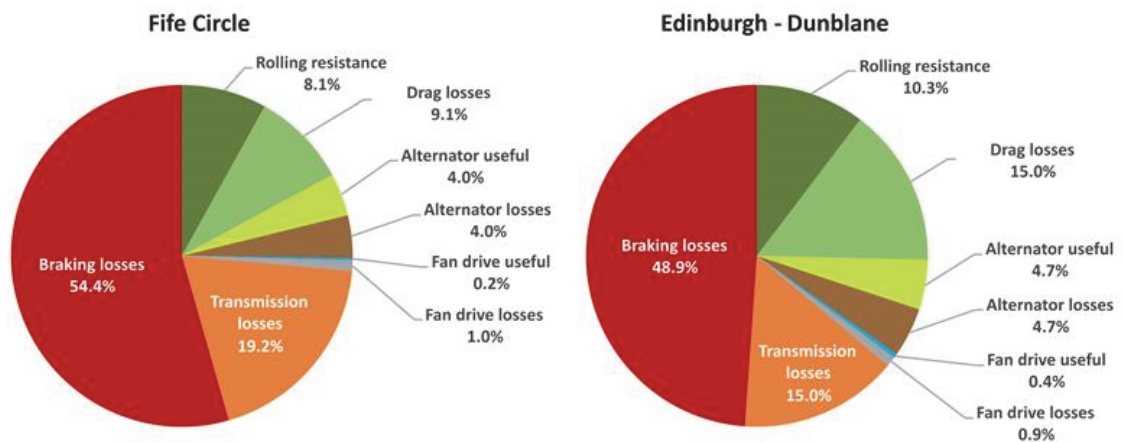

Figure 1: Energy use for Class 158 DMU, Wheals [2]

The current hydrodynamic transmissions have extremely poor efficiency at low speeds. Braking energy is entirely wasted. The engines are often operated in unfavourable parts of their operating map - where efficiency is poor and emissions are high.

If a transmission could be designed that recovered braking energy, whilst simultaneously eliminating much of the transmission loss and allowing the engine to be run at the most efficient operating point, then the fuel economy of the DMU might be significantly increased. 
Hydrostatic transmissions for railway vehicles have been investigated in the past. In 1981 British Rail built a prototype diesel powered railcar with a hydrostatic transmission, see: Tunley [3]. Conventional, readily available, hydraulic pumps and motors were utilised to provide a low-cost system. However the poor part-load efficiency of these machines meant that there was no significant improvement when compared to hydrodynamic transmissions commonly used with diesel powered railcars. Parallel-hybrid diesel railcars with hydrodynamic power transmissions using a hydrostatic recuperation system have also been investigated. Simulation results based on real route data showed significant fuel savings can be made, see Kache [4].

Artemis Intelligent Power Ltd. has developed a new class of hydrostatic pumps, motors and transmissions using a technology known as Digital Displacement ${ }^{\circledR}$ (DD). Fluid commutation and displacement control are achieved by solenoid actuated valves under the command of a microprocessor, rather than mechanical means. Digital Displacement Pumps (DDPs) have a single solenoid actuated low pressure valve and can generate a flow proportional to an electronic demand signal with a response time of the order of several milliseconds: Rampen [5]. These are an appropriate means to controllably transfer power from a diesel engine. Digital Displacement Pump Motors (DDPMs) also have a solenoid actuated high-pressure valve and, as a result, can reverse the flow through the machine, whilst also reversing the power on the shaft, Rampen [6], these are useful for regenerative applications where power flow can reverse - such as in vehicle braking. A third implementation of the machine, the Digital Displacement Wheel Motor (DDWM) is similar to the DDPM but has more complex valving between the high-pressure manifold and the cylinders in order to allow the machine to start under load in either direction. DD vehicle transmissions were first developed by Caldwell [7]. The high efficiency, power-density and excellent controllability makes DD transmissions advantageous in many applications where conventional hydrostatic machines are currently used, but also in new areas and at larger power levels, e.g. offshore wind turbines, hybrid vehicles and rail transmissions, see Taylor [8].

This paper considers a novel powertrain for self-powered rail vehicles based on DD technology. Compared to existing DMUs, the new approach can significantly reduce energy consumption and lower $\mathrm{CO}_{2}$ emissions. It also has the potential to reduce journey times through faster acceleration, which may facilitate the interoperability of diesel vehicles on electrified routes.

DD hydraulic machines are used to form a highly efficient and controllable hydrostatic transmission, linking the engine output to the wheels. The infinitelyvariable gear ratio allows the engine to always operate at speeds that correspond to optimal points on its fuel map, thus maximising its fuel efficiency. DD machines are inherently reversible, eliminating the need for separate reversing gear.

In addition to improved efficiency, the system allows braking energy to be captured. This energy is stored in on-board gas accumulators, and is reused to accelerate the vehicle. Since this captured energy can also supplement the power available from the engine, faster acceleration is possible, and consequently journey 
times can be reduced. The accumulators are effectively gas springs - charged with nitrogen. They are a standard means of energy storage, regulated by the Pressure Equipment Directive, and conventionally used on vehicles for many purposes.

Departing from stations, the engines can be shut off, resulting in much less noise and eliminating emissions in stations - as is already achieved by electric trains.

Hydraulic transmissions can aggregate power from multiple sources. This allows conventional diesel engines, such as those built in large numbers for lorries and offroad machinery to be used in combination to replace the conventional large, expensive, and bespoke engines currently found in DMUs. The engine substitution has several advantages beyond cost. The engines can be operated singly or together to match the instantaneous power requirement of the vehicle. The shutting down of an engine significantly reduces the parasitic loss of the powertrain at part-load. Two engines also provide parallel reliability. Finally, these commercial engines have been developed to the most recent emission standards and so are much cleaner than the large engines they replace.

The system may lend itself to retrofits to existing DMUs, many of which have remaining lives that are expected to be over ten years.

This paper follows the process of specifying, modelling, designing, building and testing a DD driveline suitable for a DMU.

\section{Method}

\subsection{Feasibility study}

The study was designed to specify the powertrain for a hypothetical new generation of two-car commuter trains based on the UK Class 156 DMU, for which design parameters such as vehicle dimensions, mass and resistance coefficients were provided.

Artemis began by looking at series and parallel hydrostatic transmissions for the vehicle powertrain. The series architecture was selected as this offered the best fuel consumption reduction in simulations, while also providing flexible packaging as there was no need for a mechanical driveshaft to the bogie - a major advantage in a new-build concept. The parallel architecture was noted to be more suitable for retrofit on vehicles with an existing mechanical drivetrain. Next, we considered the number of engines, pumps and hydrostatic motors. Using two smaller engines was found to give a lower fuel consumption than a single engine, as one engine could be shut down when not needed. Two driven axles per vehicle (out of four) was found to be optimal considering wheel-adhesion/starting acceleration, mechanical packaging and cost. The final concept was a hydrostatic transmission using two separate engines, each driving a DD pump, and two bogie-mounted DDWM hydraulic motors, with gas accumulator storage to recover braking energy. The original AEC Railcar, as operated 
by the GWR from the 1930s until 1962, had a very similar multi-engine configuration, albeit with a Wilson pre-selector gearbox driving each wheel set, see Daniel [9]

The next step was to optimise the size and type of the individual components. MATLAB/Simulink was used to build time-domain simulations of the proposed DD Hybrid vehicle and, also, of a baseline DMU. This allowed vehicle performance to be assessed and enabled quantification of fuel savings. The hydraulic transmission was modelled as a 1 degree of freedom system, with flow input by the DDP and input/output from the DDPM (depending on whether it was pumping or motoring). The lumped system was modelled with a linear compliance when the gas accumulator was isolated from the system. The gas accumulator itself was modelled using the Benedict-Webb-Rubin (BWR) equation of state, which relates gas pressure to its temperature and specific (molar) volume through eight coefficients. This gives a more accurate representation of the thermal losses of the gas, including the foam fraction which was used in the accumulators to improve their thermal behaviour.

Both 'backward-facing' and 'forward-facing' techniques were used; Figure 2 shows a simplified diagram of the forward-facing model.

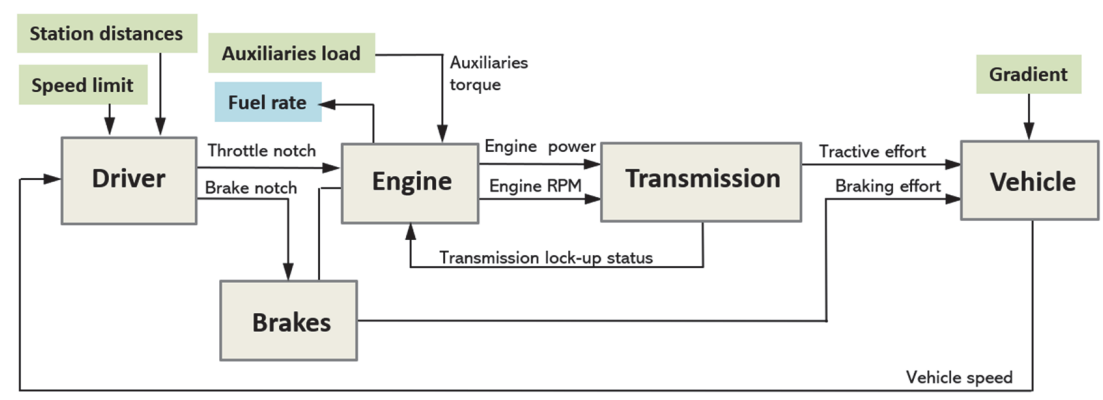

Figure 2: Baseline Simulink model overview

In parallel with the simulation work Artemis carried out a packaging study to show how the system might be installed under the floor of the target vehicle, leaving the space above free for passenger accommodation. Figure 3 shows the conceptual underside layout of the vehicle, with the powered bogie at the left and two engine rafts located adjacently with the energy storage accumulators to the right.

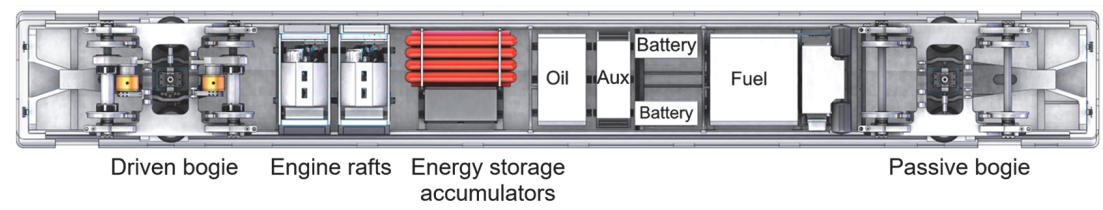

Figure 3: Concept for DMU conversion 
Figure 4 shows the concept for the powered bogie: it is equipped with a $480 \mathrm{cc} / \mathrm{rev}$ DDWM for each wheelset, driving through a hollow shaft gearbox and a quill shaft, so that the motor is fully suspended and unsprung mass is minimised.

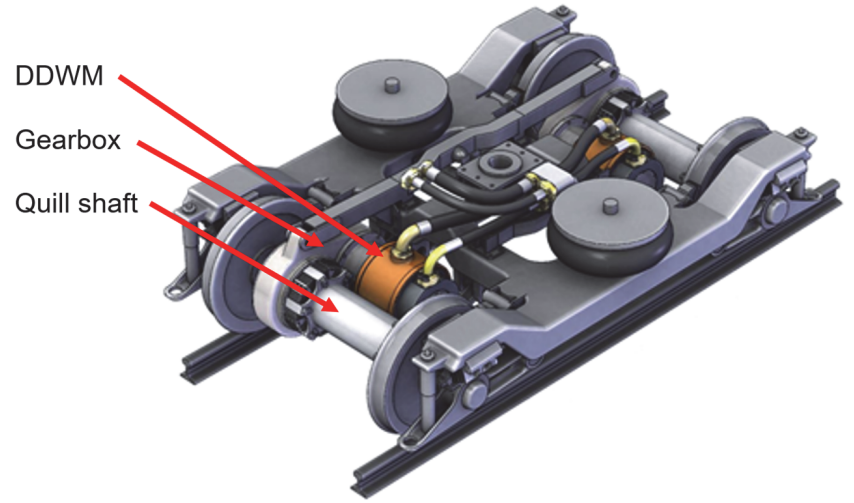

Figure 4: Concept design of DD hydrostatic drive system fitted to a DMU bogie (identical drivetrain on front and rear wheelsets)

The outcome of the study was that the system could be feasibly packaged in a typical DMU vehicle and would offer the following advantages:

- Significant fuel savings (up to $38 \%$ on the sample routes studied)

- Improved acceleration performance for faster journeys

- Departure from stations without engine emissions

- Elimination of brake wear

- Applicable to non-electrified routes

\subsection{Demonstration stage}

After successful completion of the feasibility study, the project progressed to vehicle demonstration with the aim being to show the full series hybrid system operating in a rail vehicle. Although the hybrid DD powertrain was ultimately intended to be fitted on DMUs, there were none available in the UK. Instead a trailer car, from an Intercity 225 (Mark 3 DVT), loaned by Chiltern Railways was used. This vehicle had similar mass and overall dimensions to a DMU vehicle. J C Bamford Excavators Ltd (JCB) provided two of their $129 \mathrm{~kW}$ ecoMAX engines for the primary power units. Figure 5 shows the modified DVT railcar undergoing track testing.

The forward-facing simulation models used in the feasibility study were further refined and were used to develop the control system for the vehicle. Model-based design in Matlab-Simulink allowed the same control code, as developed in the simulation model, to be deployed for the actual vehicle control on XPC hardware.

Practical work started with test rig-based development of some core components for the DDWMs, followed by commissioning and testing of the completed DDWMs and DDPs. Laboratory testing finally progressed to a large half-vehicle test rig with 
one engine-driven DDP driving a DDWM which was loaded to simulate half of the inertia and resistance of the carriage. A 365 litre gas accumulator bank for energy storage was also connected, pre-charged to 150 Bar. This allowed the control system to be extensively developed and tested ahead of work on the vehicle.

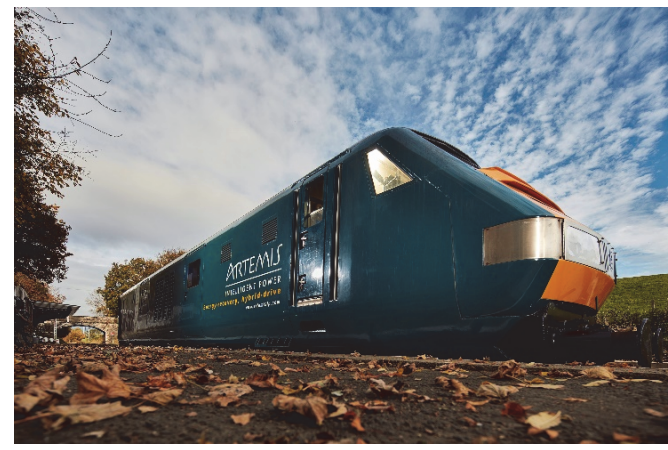

Figure 5: DVT rail car track testing

As with the DMU concept in Figure 3, the converted DVT had the hydraulic accumulators mounted below the floor and the two DDWMs installed into one of the bogies (front bogie of the DVT), as shown in Figure 6. For reasons of cost and accessibility, for the prototype vehicle the engines and DDPs were installed inside the body of the trailer. A new driver station was added at the rear of the vehicle to allow bi-directional operation on the test track. Display screens were added to each cab to show important parameters of the series hybrid transmission whilst operating the railcar; these were driven by a Dewesoft data acquisition system. Two CANbus networks were used to connect the top-level controller, control units for the two engines, the two DDPs, two DDWMs, driver controls and auxiliary inputs and outputs.

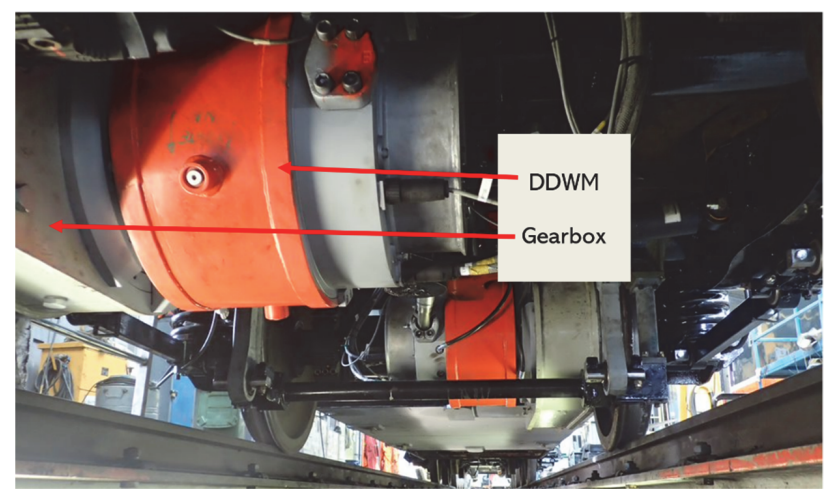

Figure 6: Underside of DVT showing power bogie and hydraulic motors (red components) 
Figure 7 show the power flow connections between the various parts of the drive system. Figure 8 shows the layout of the powertrain of the modified DVT railcar. Figure 9 shows the hydraulic system layout.

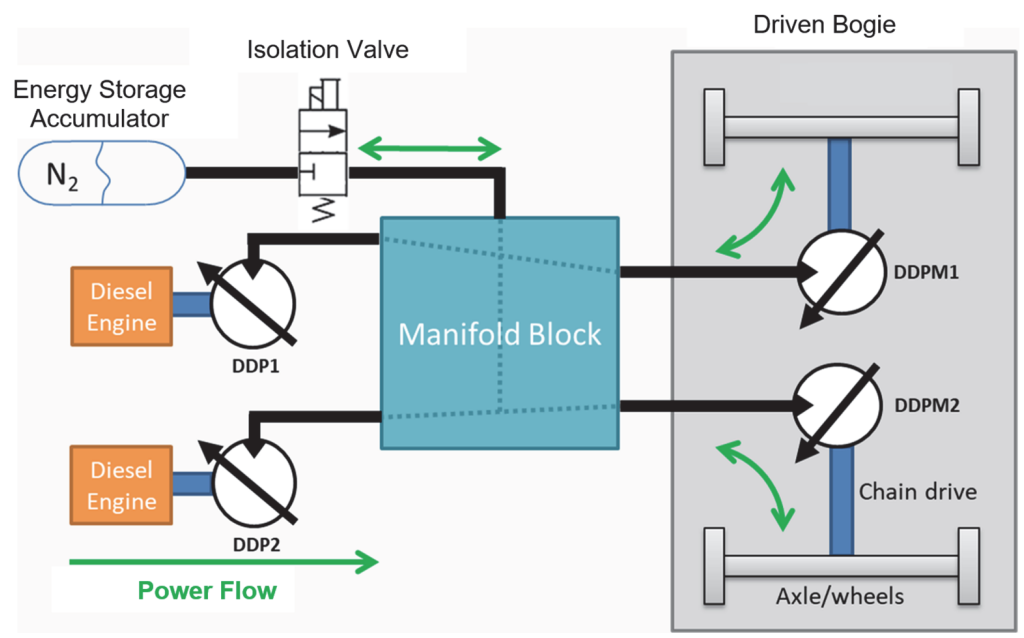

Figure 7: Power flow schematic showing hydraulic and mechanical drive connections to and from diesel engines, energy storage and wheels

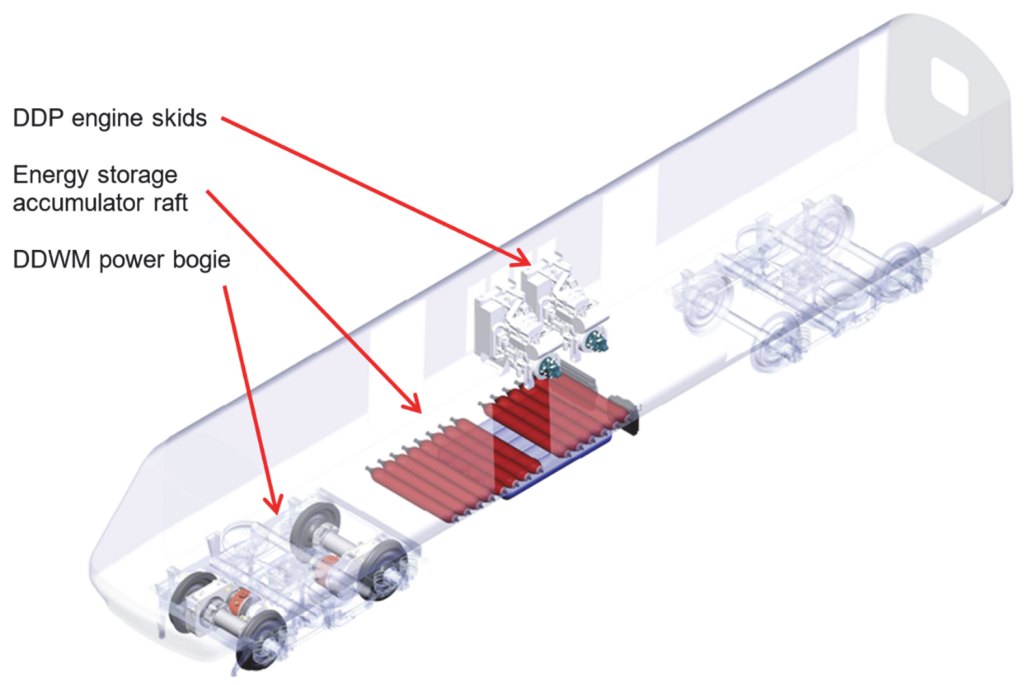

Figure 8: DVT powertrain layout 


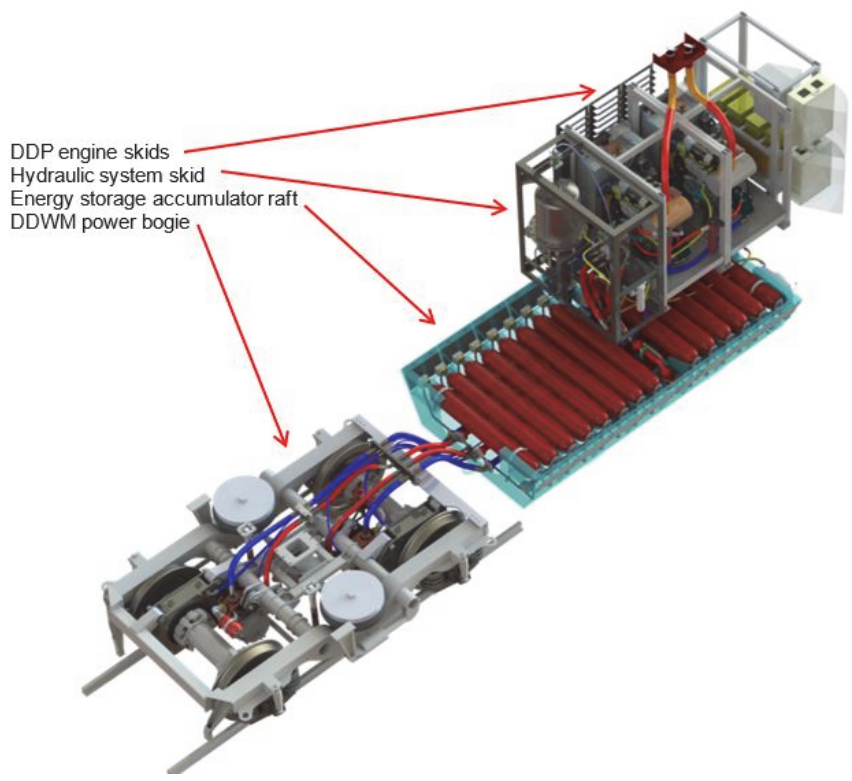

Figure 9: Layout of the hydraulic system

\section{Testing and Results}

There were two major phases in the testing programme: static test on a purpose-built half-driveline system rig and actual vehicle tests with on-board data acquisition.

The former was used as a means of verifying and calibrating the simulation models that had been developed earlier. Once the models could be trusted, they could be used to provide simulations of the DVT railcar which could, in turn, be compared with the actual results from the vehicle. Given that the test conditions of the actual railcar were dictated by the track layout (speed restrictions, changes of gradient, etc.) it was not possible to directly produce actual performance results that could be applied to a similar DMU. But by confirming the performance predictions of the model, in terms of vehicle dynamics and instantaneous fuel consumption on the test track with known gradient profile, the validated model itself could then be used to predict DMU performance with reasonable confidence.

The half-driveline system consisted of one engine driving one DDP, in turn powering one DDWM driving a dynamometer which simulated the vehicle kinematics. The measured torque of the DDWM was applied to a real-time model of the train kinematics, including inertia, rolling and aerodynamic loads. The model outputted a simulated vehicle speed; the electric motor shaft was then controlled to 
this speed, with full four quadrant control, thus allowing development of the control system ahead of fitting the driveline to the vehicle. The rig architecture is shown below in Figure 10.

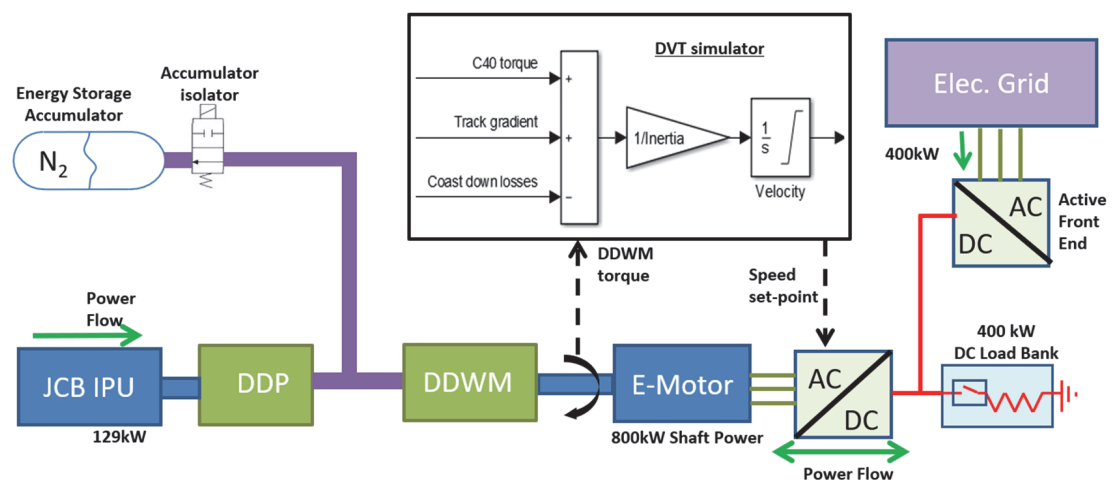

Figure 10: Signal and power flows for the static, half driveline, system test rig

The efficiency of the transmission is defined as the wheel input power divided by the engine output power. The wheel input power was measured directly by the high accuracy torque transducer installed between the DDWM and the electric motor. The shaft speed was also directly measured. However, directly measuring engine output power was not possible; the DDP was coupled to the engine bellhousing, so there was no space to fit a torque transducer. Instead, the instantaneous engine output torque was indirectly calculated using experimentally validated data of the DDP.

This was produced on a separate test rig, which had torque and flow measurement capability, and which allowed a comprehensive model of the DDP to be calibrated. The structure of this model has been described previously by Caldwell [5]. Pressure, speed and displacement fraction were used as input to the model, shaft torque and fluid flow formed the output. Losses and efficiency were thus calculated. Figure 11 shows the comparison of experimental and model data for the DDP.

In summary, for the railcar system efficiency model, the transmission output power was directly measured, and the transmission input power was indirectly measured, using measurements from the transmission rig in addition to a machine model calibrated on a separate test rig.

All torque transducers used had a total accuracy of $\pm 5.5 \mathrm{Nm}$, pressure transducers had a total accuracy of \pm 0.55 bar and encoders had a resolution of 2048 pulses per rev.

The overall driveline efficiency on the test rig is plotted as the light grey solid line in Figure 12, as a function of vehicle speed. Due to test rig limits, the maximum possible speed was $95 \mathrm{~km} / \mathrm{h}$. 

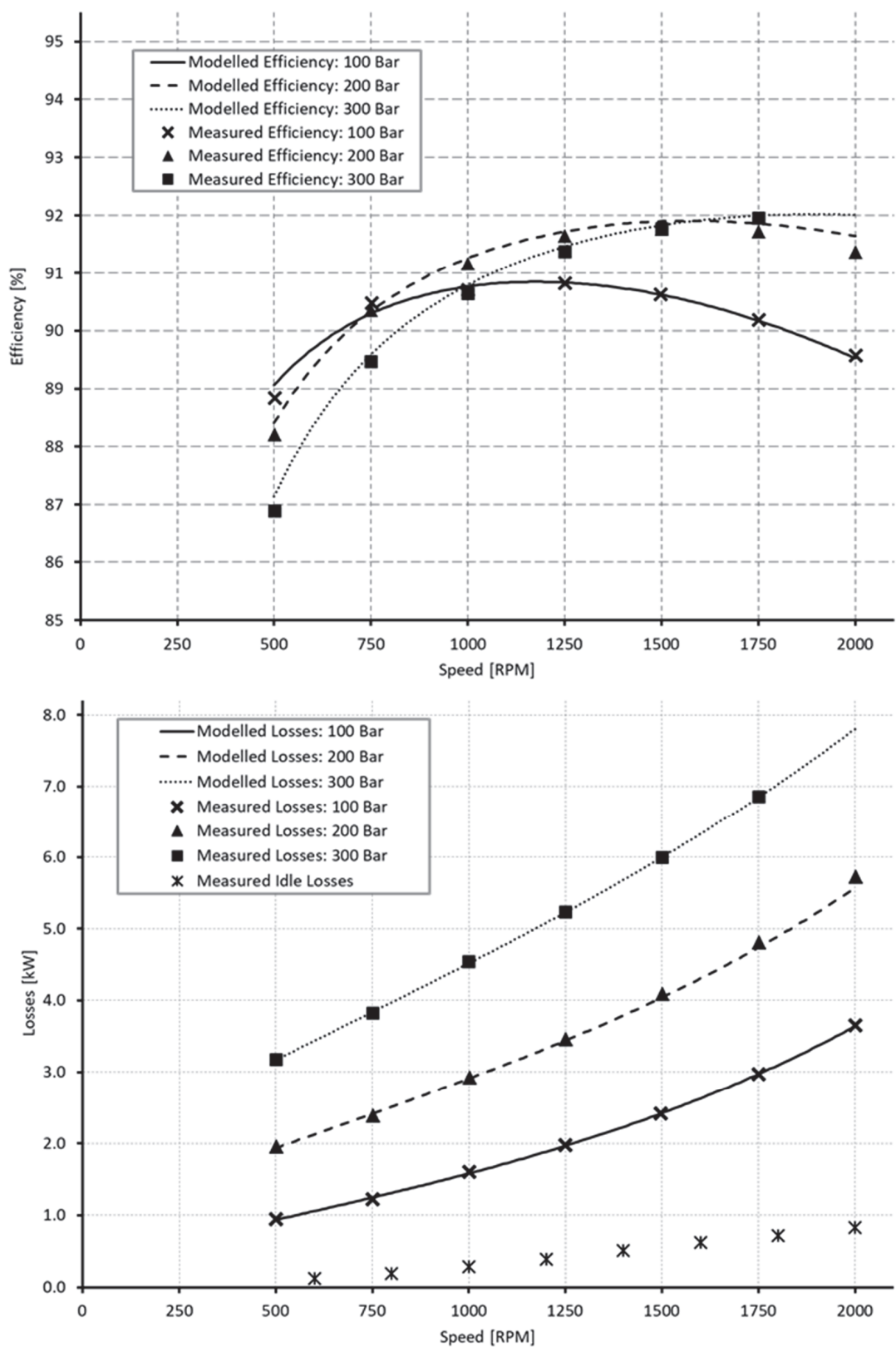

Figure 11: Experimental and modelled efficiency of the DDP 


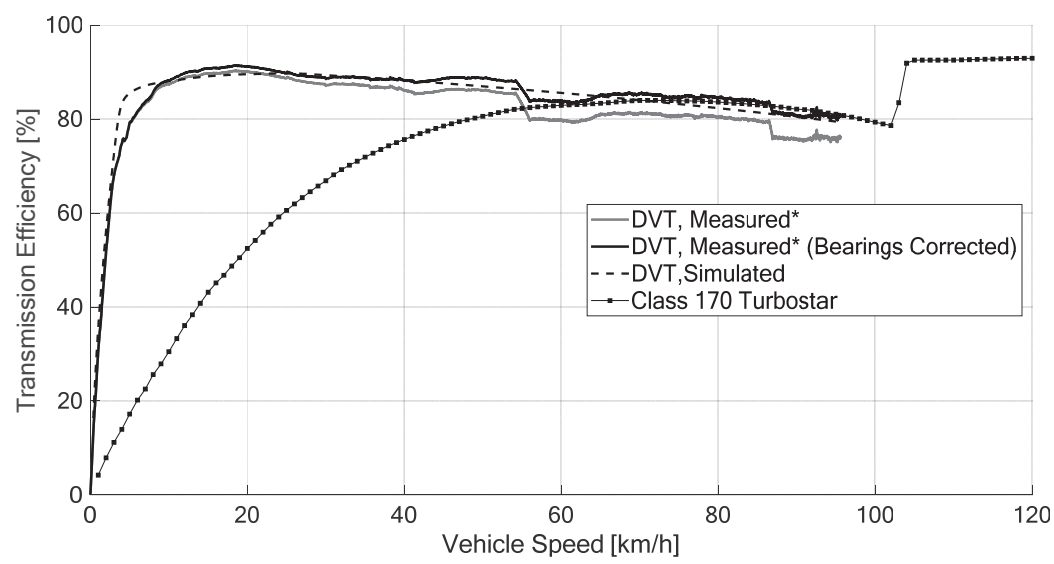

Figure 12: Efficiency data for the DVT railcar and Class 170 DMU

The test rig measured efficiency closely matches with the simulated efficiency (dashed line). The test rig efficiency is slightly lower than the simulation prediction at higher speeds; this is due to the need to advance the timing of the valves of the DDWM, for stable operation, which in effect shrinks the machine's flow capacity, but not the losses.

This can be compared to the hydrodynamic transmission from a typical Class 170 DMU (dotted line), where efficiency barely exceeds $80 \%$ below $90 \mathrm{kph}$, due to the high churning losses in the torque converter. The hydrodynamic transmission jumps to a much higher efficiency at approximately $100 \mathrm{kph}$, when it switches to a fixed mechanical gear.

Further development of the DDWM machine design has shown that significant improvement can be made to the efficiency of the transmission by ensuring that the shaft bearings are partially, rather than fully, flooded. The effect of partially flooded vs. fully flooded bearings was measured in a separate test, and the solid line in Figure 12 is the test rig efficiency adjusted to show the effect of this improvement.

The apparent 'steps' at $55 \mathrm{kph}$ in the measured data are a result of the DDWM motor flow command being quantised (i.e. the control signal to the DDWM is stepped). These control steps were used to avoid oscillation on the low-pressure side of the test rig. The actual DVT could use a 'non-quantised' flow command (as in the simulation), since the low-pressure manifold design in the actual train is superior to that of the test rig.

Finally, the test rig setup allowed the round-trip efficiency of the hydraulic Kinetic Energy Recovery System (KERS) to be demonstrated. The simulated rolling and drag coefficients of the railcar were set to zero, to represent a purely inertial 
vehicle. By accelerating and decelerating the railcar only using the energy storage, the KERS could be shown to consistently re-use the vehicle's braking energy with a round-trip efficiency of above $82 \%$. The percentage values in the top graph of Figure 13 indicate the percentage of energy lost between each cycle. The efficiency could be improved if, as mentioned above, the design of the roller bearings was improved with a simple modification. This KERS result is a significant and notable improvement on the efficiency of modern electrical vehicles, whose KERS efficiencies achieve $70 \%$ under the most favourable circumstances, see Boretti [10].
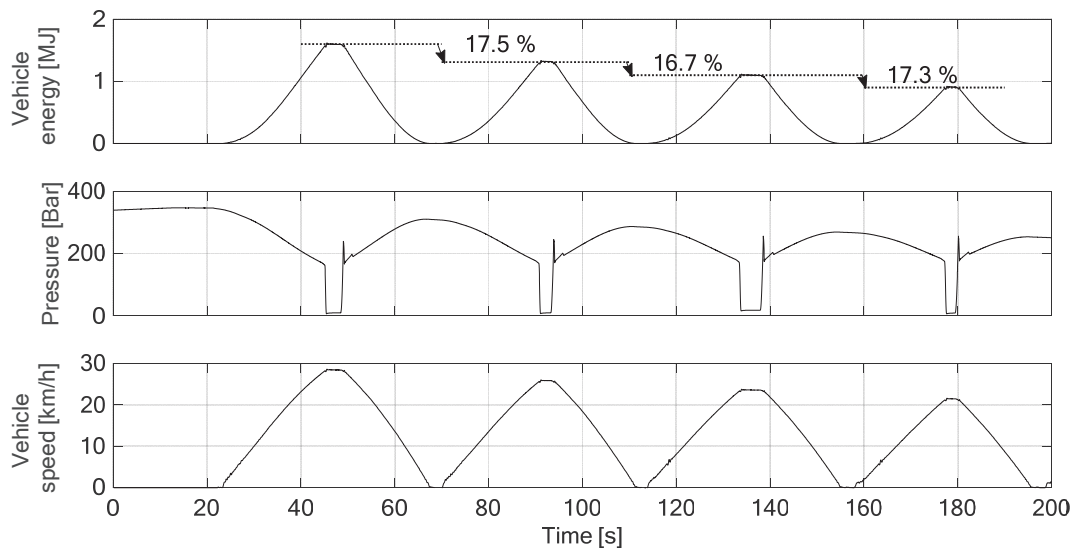

Figure 13: Measured round-trip efficiency of hydraulic KERS with simulated inertia

Having confirmed the transmission, in terms of component behaviour, control commissioning and simulation model validation on the static rig, the next step was to install the full driveline transmission into the railcar and thus prove the concept in operation, showing that it convincingly matched the simulated behaviour on a real track. The converted railcar was tested on a heritage railway track in Bo'ness, Scotland. Several modes of operation were successfully tested including:

1. IVT mode: Infinitely Variable Transmission mode, in which the flow from the engine-driven DDPs supplies the bogie-mounted DDWMs. The effective transmission ratio is infinitely variable; the engine speed could thus be controlled to its most efficient operating point for each power level.

2. Pressure Relief Valve Braking: The DDWMs were operated to pump over a pressure relief valve at 350 bar, thus giving maximum braking torque, without any storage. Torque could be modulated by choosing the rate at which cylinders were being actuated to pump. A cooling system dissipated the heat generated in the oil. The vehicle was able to brake from $25 \mathrm{mph}$ to standstill in $130 \mathrm{~m}$ using hydraulic braking alone, which is well within the GM/RT2045 standard of $244 \mathrm{~m}$ for the railcar's mass. 
3. Regenerative Braking: The DDWMs were operated to pump into the gas accumulators, thus capturing the braking energy which, on a conventional vehicle, is wasted as heat in the brake discs.

4. Eco-mode: The energy stored in the accumulators was used to supply the DDWMs, meaning the engines could simply idle, or even be switched off as the vehicle accelerated. This mode is particularly useful at start-up, while the train is in the station. Once the accumulators were depleted, the control system automatically switched to IVT mode.

5. Boost mode: Both the energy stored in the accumulators and the engines were used together to supply the DDWMs, meaning greater power available at the wheels, and higher acceleration rates.

The timeseries plots from experimental runs at Bo'ness in Figure 14 show operation encompassing the following modes: IVT mode (sections A and D), Regenerative Braking and Eco-mode (section B), PRV braking (section C), ECO mode (section D), IVT mode again (section E) and finally a short period of Regenerative Braking (section F).
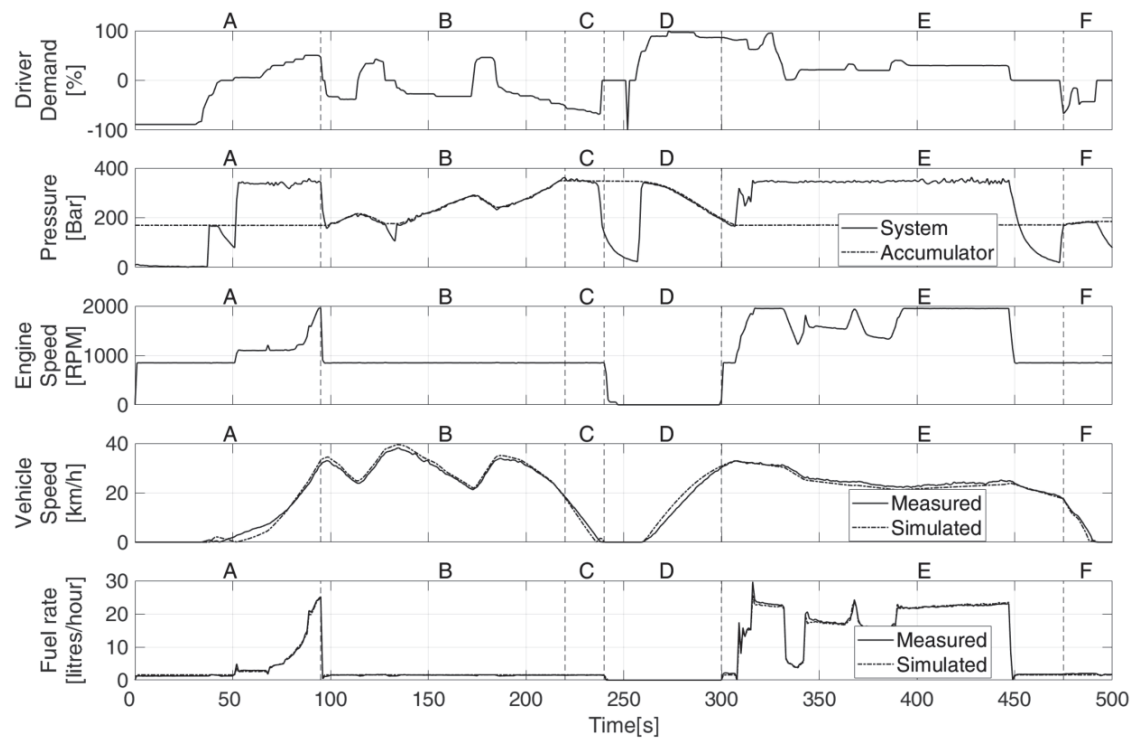

Figure 14: Times series plots showing DVT railcar operation, comparing measured and simulated results

Figure 14 shows driver demand ranging from -100\% (full braking) to $100 \%$ (full acceleration). When using brake regeneration or eco-mode, the accumulator is connected to the DDWM high pressure line. The accumulator fill state determines its 
actual pressure, which the DDWM will match in order to move fluid in and out of it. For example it can be seen in section B that the accumulator pressure increases as the railcar decelerates and stores both energy and fluid in the accumulator, and decreases as it accelerates and the accumulator empties. When the railcar is at rest, or is using IVT mode, the accumulator is automatically isolated using a solenoid valve. In this case, the DDWM pressure is controlled by the system controller, to the optimal setpoint.

The engine speed is controlled dynamically so it is always at the optimal operating point for a given power demand. For example, in section A when departing in IVT mode, the engine runs at 1050 RPM, just above idle speed. This is in contrast to a hydrodynamic torque converter transmission, where the engine operates at around 1900 RPM at departure. The engine speed goes to zero at section D; both engines were automatically switched off not only while the vehicle was stationary, but also while it accelerated using stored energy, up to the point when the energy store was depleted.

A key result of the project is displayed in the bottom two plots of Figure 14. These show a very good match between simulation and measurement for both vehicle speed and engine fuel rate. The average error over this 500 second example is $5.4 \%$ for vehicle speed, and $3.7 \%$ for fuel rate.

Discrepancies in vehicle speed, for example at the initial acceleration in section A, are most probably due to inaccuracies in the test track grade profile data, where resolution is at certain points as low as $2.6 \mathrm{~km}$. Much of the track gradient profile was approximately 1 in 95 , so a change in potential energy had a dominant effect on the railcar during these tests. Inaccuracy in the measured railcar mass and measured transmission efficiency rig were less important. Discrepancies in engine fuel rate are most apparent at higher engine speeds. The most obvious reason for this is the estimation of auxiliary loads - alternator, compressor, fan, and circulation oil pump. Although each of these estimates were based on manufacturer's data, the resolution of the power curves, and their sensitivity for example to temperature (of the alternator) or air flow restriction (of the fan) meant that they are likely to deviate from the actual load on the engine, especially at higher speeds where their torque is greater.

The close overall agreement between the measured railcar performance and fuel rate gave confidence in using the simulation model as a tool for predicting the performance and the fuel saving of an actual DMU, fitted with the DD hydraulic system, over a real-world drive cycle. This meant that it could be reliably used to compare a modelled DD driveline with a conventional one in a baseline DMU.

A forward-facing simulation was used for the drive cycle comparison. A closedloop simulated driver provided inputs to the model (throttle and braking commands), driving three routes on the rail network. A forward-facing Simulink model was used for two reasons:

- Station distances and track speed limits were provided for the route but not a speed profile that was to be followed. 
- The forward-facing simulations allow different control strategies to be tried in order to quantify the effect on fuel use and vehicle performance. A backwardfacing simulation would not provide this flexibility.

In the forward-facing model, the simulated driver has the following behaviour:

- Follows a route with specified speed limits and station locations, and track gradients.

- Requests maximum acceleration when below the speed limit.

- Requests an acceleration or braking percentage to cruise at constant speed when a speed limit is reached.

- Slows down in anticipation of speed limits and stations.

The simulation proceeds through time-steps of typically 50 milliseconds. At each time-step it outputs vehicle speed and fuel rate. Other signals such as instantaneous tractive-effort and engine power can be output for diagnostics and analysis. The engine provides power for auxiliary 'hotel' loads as well as traction.

In 2010 Artemis worked with First ScotRail to study the potential benefit of using Digital Displacement ${ }^{\circledR}$ hydraulic technology in commuter rail applications. A Class 158 DMU was fitted with sensors and data-logging equipment during operation on two Edinburgh routes, Figure 1. By using our knowledge of the similarities between the Class 170 and Class 158, the baseline Class 170 model was been calibrated against data recorded during this project. Table 1 compares the parameters of both the Artemis DD DMU and the Class 170 (in both cases modelling one car). Note that the Class 170 has $18 \%$ greater engine power, and is approximately $10 \%$ heavier, per car. Note also that this simulation uses two DDWMs of size $720 \mathrm{cc} / \mathrm{rev}$, as opposed to the prototype DVT which used two DDWMs of size $480 \mathrm{cc} / \mathrm{rev}$ both with gear ratios of 1:1.64. The prototype train DDWM size was limited by current availability of machines. The simulated train had larger DDWMs in order to satisfy the 'worst case' tractive effort requirements to move the simulated DMU railcar (maximum number of passengers, 'gale' winds of $50 \mathrm{mph}$ and $1 / 50$ gradients, as advised by rail industry professionals).

Three separate routes were modelled. This paper presents the results of just one of these routes, Maidenhead to Marlow. Table 2 gives a statistical summary of the route. It has short distances between stations, and a low maximum speed limit, and therefore is a route where brake energy recovery and higher transmission efficiency at low speed are particularly advantageous.

The Artemis DD DMU simulation uses a total accumulator capacity of 660 litres, with a gas pre-charge of 200 bar. This is a compromise between maximising brake energy capture and minimising cost, weight and size. 


\begin{tabular}{|l|l|l|}
\hline & Artemis DD DMU & Class 170 \\
\hline Engine & Two JCB Tier 4F & MTU 6R 183 RD13 H \\
\hline Rated Power & $2 \times 129 \mathrm{~kW}$ & $315 \mathrm{~kW}$ \\
\hline Auxiliary Load & $22.5 \mathrm{~kW}$ & $22.5 \mathrm{~kW}$ \\
\hline Transmission & $\begin{array}{l}2 \times 96 \mathrm{cc} / \mathrm{rev} \text { DDPs } \\
\text { and } 2 \times 720 \mathrm{cc} / \mathrm{rev} \text { DDWMs }\end{array}$ & Voith T211 re3 \\
\hline Axle drive gear ratio & $1.64: 1$ & $2.47: 1$ \\
\hline Mass of one car & 42 tonnes & 46.7 tonnes \\
\hline Coast down coefficient ' $A$ ' & $938 \mathrm{~N}$ & $938 \mathrm{~N}$ \\
\hline Coast down coefficient ' $B$ ' & $8.5 \mathrm{~N} / \mathrm{m} / \mathrm{s}$ & $8.5 \mathrm{~N} / \mathrm{m}^{\prime} / \mathrm{s}$ \\
\hline Coast down coefficient ' $C$ ' & $2.9 \mathrm{~N} / \mathrm{m}^{2} / \mathrm{s}^{2}$ & $2.9 \mathrm{~N} / \mathrm{m}^{2} / \mathrm{s}^{2}$ \\
\hline Maximum speed & $160 \mathrm{kph}$ & $160 \mathrm{kph}$ \\
\hline
\end{tabular}

Table 1: Comparison of Artemis DMU and Class 170 railcars. (All values are used are 'per car')

\begin{tabular}{|l|c|}
\hline & Maidenhead to Marlow \\
\hline Mean Station Distance & $2.9 \mathrm{~km}$ \\
\hline Max Station Distance & $4.3 \mathrm{~km}$ \\
\hline Min Station Distance & $2.4 \mathrm{~km}$ \\
\hline Number of stops & 9 \\
\hline Total distance & $23.2 \mathrm{~km}$ \\
\hline Total height climbed & 55 metres \\
\hline Maximum speed limit & $130 \mathrm{kph}$ \\
\hline
\end{tabular}

Table 2: Summary information for the Maidenhead to Marlow route

Figure 15 shows sample results of approximately ten minutes operation from the forward-facing simulation of the Maidenhead to Marlow route, using the Class 170 . The middle plot clearly shows the simulated driver moving the notched torque and braking control. In the lower plot, the difference between the engine power and wheel power is largely accounted for by the assumed steady $22.5 \mathrm{~kW}$ auxiliary hotel load in each DMU railcar. Further work could involve more accurate modelling of these loads, and their dependency on conditions such as ambient temperature.

Figure 16 compares the full throttle accelerations of the Artemis DD DMU (simulated) with the Class 170 (recorded data from a real train). During the first 40 seconds, the DD DMU Boost mode is fastest, since it utilises both engine power and accumulator power. Eco-mode uses only accumulator power initially. In both cases, once the accumulator storage is depleted, Boost mode and Eco mode will be running only on engine power. Both modes result in faster initial acceleration than the Class 170. 

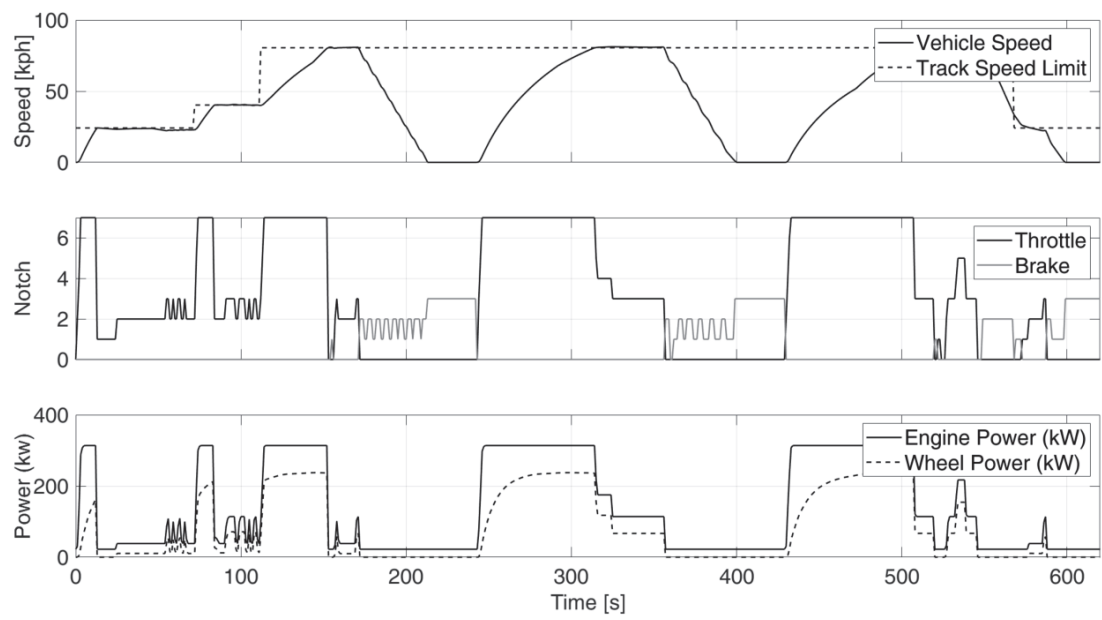

Figure 15: Class 170 Baseline DMU simulation (section of Maidenhead to Marlow)

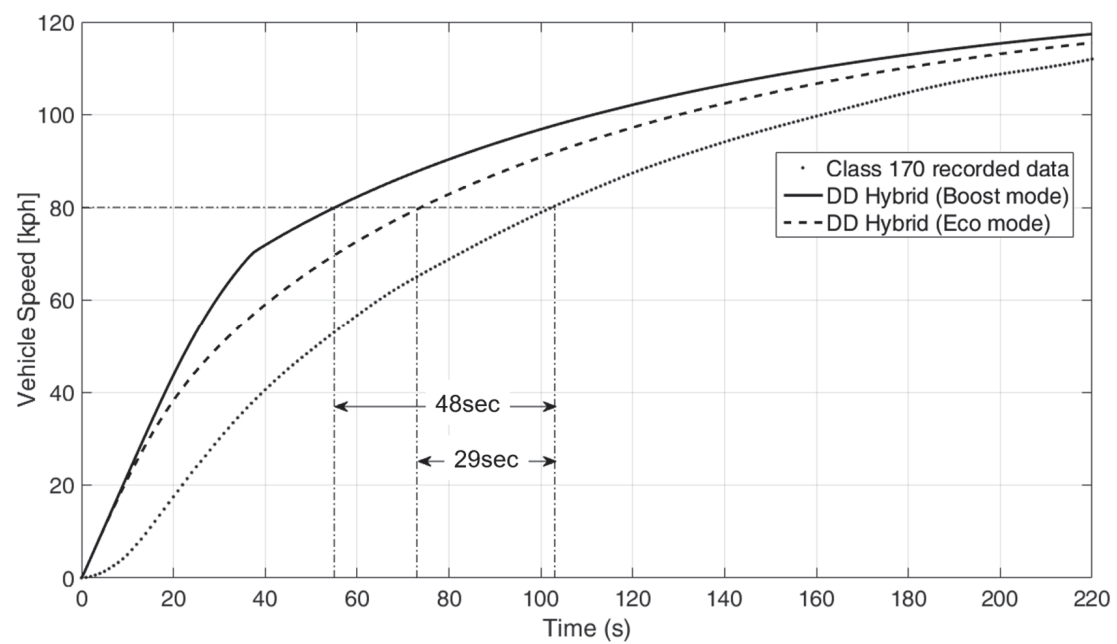

Figure 16: Comparison of initial acceleration for the DD DMU in Eco mode and Boost mode, and recorded data of a class 170

Table 3 compares the Class 170 DMU to the Artemis DD DMU in both Eco mode and Boost mode, driven over the Maidenhead to Marlow route. The route times can be compared with the timetable value of 41.5 minutes. 


\begin{tabular}{|l|c|c|c|}
\hline & Class 170 & DD DMU(Eco) & DD DMU(Boost) \\
\hline Average Engine BSFC [g/kWh] & 222 & 206 & 205 \\
\hline Engine Energy Used [MJ] & 223 & 148 & 160 \\
\hline Fuel Used [litres] & 16.5 & 10.2 & 10.9 \\
\hline Fuel saving [\%] & - & 38.2 & 33.7 \\
\hline Route Time [minutes] & 32.3 & 32.5 & 31.5 \\
\hline
\end{tabular}

Table 3: Breakdown of fuel use and vehicle performance on Maidenhead to Marlow route

A number of observations can be made with regard to Table 3:

- When the Artemis DD DMU is operating in Eco mode, fuel savings are made by three means in comparison to the Class 170 DMU: firstly, the average engine BSFC is $7 \%$ lower, meaning the engine is used more efficiently, secondly, captured braking energy is re-used, and thirdly the transmission efficiency is higher; the combination of these can result in a $38 \%$ reduction in fuel used.

- Due to the track speed limits on this route, there is little opportunity for Boost mode to be used, since the accumulator or engine alone can satisfy the DMU total power demand. This is illustrated in the by the fact that Boost mode uses only $8 \%$ more engine energy than eco-mode. In any case, a 33.7\% fuel saving is achieved, with a 48 second journey time reduction.

Other routes simulated with higher track speed limits showed much more significant journey time reductions using Boost mode. For example on the Norwich to Lowestoft route, the DD DMU was 4 minutes 24 seconds faster than the Class 170, a $5 \%$ reduction in journey time. This was in addition to saving $9.7 \%$ of fuel in comparison to the Class 170 . On the same route, Eco-mode made a $37 \%$ fuel savings and was only 1.5 minutes slower than the Class 170 .

Clearly the operator could balance the use of Eco and Boost modes in a single journey, depending on their priority between fuel use and journey time.

The energy reductions of Eco-mode are illustrated in Figure 17. Transmission loss energy is approximately halved, and $20 \%$ less energy goes into the friction brakes, since it is captured by the accumulators and re-used for propelling the railcar.

\section{Conclusions and Further Work}

\subsection{Conclusions}

This project represents the first successful application of a regenerative DD hydrostatic transmission in a rail vehicle driveline. The incorporation of the propulsion system into an existing rail vehicle has been achieved without significant structural change. 


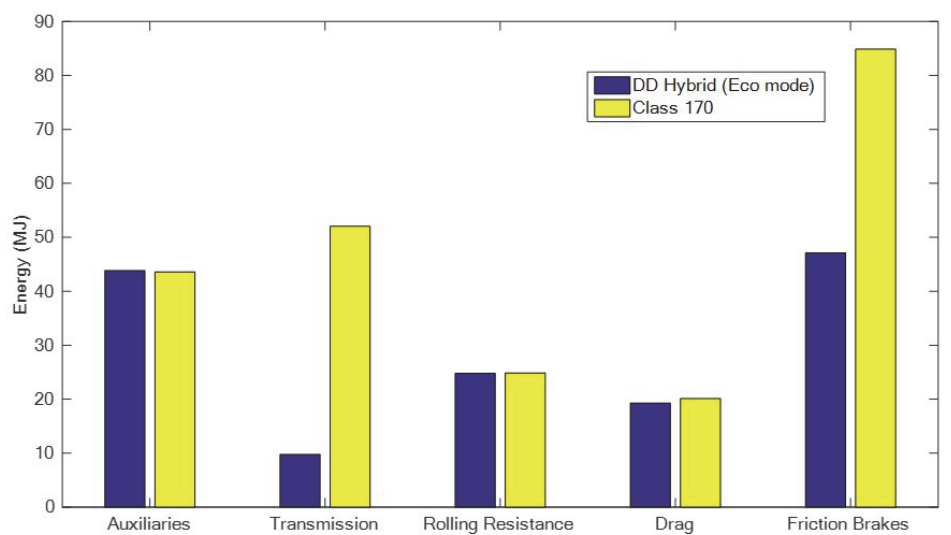

Figure 17: Breakdown of engine energy use on Maidenhead to Marlow route

The inclusion of energy storage permits the vehicle to move into and out of stations with the IC engines turned off, thus eliminating noxious emissions in these oftenconfined spaces.

The improvement to fuel economy for a Class $170 \mathrm{DMU}$, in the range of $32 \%$ to $38 \%$, dependent on route, is a substantial operational improvement both in terms of reducing operating cost and also $\mathrm{CO}_{2}$ emissions.

The system has potential to improve the acceleration performance of DMUs to match that of EMUs through the combination of IC engine output and stored energy. The results predict journey time reductions of $5 \%$ on some routes. This can allow interoperability and increase track capacity through faster acceleration and improve customer satisfaction though faster journey times. As the DMU is independent of the electric infrastructure it can add capacity to a line, where volume traffic is being capped by electrical current demand, during peak times.

The drivers of the demonstration railcar found the continuous four quadrant control afforded by the DD transmission easy to adapt to and were very satisfied with its driveability.

The system's modular design allows for both retrofitting to existing rail fleets or new-build vehicles.

\subsection{Further work}

With a consortium in place, the next phase of this project would involve a trial of the powertrain on a train operating on a railway network, to prove reliability and measure benefits in back-to-back testing alongside the standard DMU carriage. To achieve this, an existing DMU train could be retrofitted, beginning with a single DMU railcar, followed by a complete trainset and finally a retrofit of a fleet of vehicles. 
One challenge is to find suitable vehicles that could be available for retrofitting with the new powertrain. At the time of writing the authors are investigating the feasibility of modifying a fleet of Class 314 EMU's destined to be scrapped as soon as they are withdrawn from service in the near future. These railcars, although not DMUs, could still be suitable for retrofitting with this powertrain. They already have a bogie transmission layout similar to the hydraulic transmission. The original electric motors can be swapped with DDWMs, the final drives can be modified to the required gear ratio.

\section{Acknowledgements}

The authors would like to thank the RSSB, Chiltern Railways, J C Bamford Excavators Ltd, Centa Transmissions Ltd, Hydac International GmbH, Gmeinder Getriebe and SRPS Diesel Group for their support in this work.

\section{References}

[1] F. Cignini, A. Genovese, F. Ortenzi, A. Alessandrini, L. Berzi, L. Puigi, R. Barbieri, "Experimental data comparison of an electric minibus equipped with different energy storage systems", Batteries, 6 (2), 2020, doi: $10.3390 /$ batteries 6020026

[2] J.C. Wheals, W To, J Dalby, M Vigar, J Hodgson, J Buchanan, A. Robertson, J MacPherson, J Taylor, Artemis IP; W Lanoe, M Heaton, "Viable flywheel system for rail", The Stephenson Conference - Research for Railways, London, UK, 2015.

[3] J.D. Tunley, "A hydrostatic transmission for a railway passenger vehicle", British Rail Research, Derby, C155/81, IMechE, 1981.

[4] M. Kache, "Investigating an all-hydraulic for diesel-hydraulic rail cars", Eur. Transp. Res. Rev., 6:181-189, 2014.

[5] W.H.S Rampen, S.H. Salter, "The Digital Displacement ${ }^{\circledR}$ Hydraulic Piston Pump", Proc. 9th International Symposium on Fluid Power, BHR Group, Cambridge, UK, STI, pp. 33-46, April 1990.

[6] W.H.S. Rampen, J. Almond, S. Salter, "The Digital Displacement ${ }^{\circledR}$ Pump/Motor Operating Cycle: Experimental Results Demonstrating the Fundamental Characteristics", 7th Bath Intn'l Fluid Power Workshop, Innovations in Fluid Power, Bath, UK, Sept. 1994

[7] N. J. Caldwell, "Digital Displacement Hydrostatic Transmission Systems", PhD Thesis, The University of Edinburgh, UK, 2007.

[8] J. Taylor, W. Rampen, D. Abrahams, A. Latham, "Demonstration of a Digital Displacement Hydraulic Hybrid Bus", JSAE Annual Congress, Yokohama, Japan, 2015.

[9] J. Daniel, Great Western railcars, [online] Available at: [http://www.greatwestern.org.uk/aec1.htm], 2013 
[10] A. Boretti, "Analysis of the Regenerative Braking Efficiency of a Latest Electric Vehicle," SAE Technical Paper 2013-01-2872, 2013 